\title{
Eficácia do canabidiol no tratamento de convulsões e doenças do sistema nervoso central: revisão sistemática
}

\author{
Arnóbio Barros Santos ${ }^{\mathrm{a}}$, Jackelyne Roberta Scherf ${ }^{\mathrm{b}}$, Rafael de Carvalho Mendes ${ }^{\mathrm{a}^{*}}$ \\ a Faculdade de Medicina Estácio de Juazeiro do Norte, Juazeiro do Norte,63040-360, Ceará, Brasil. *rafa_mendes@msn.com \\ b Faculdade de Juazeiro do Norte, Juazeiro do Norte, 63010-475, Ceará, Brasil.
}

Recebido: 3 outubro 2018 / Aceito: 1 dezembro 2018 / Publicado online: 2 janeiro 2019

\begin{abstract}
Resumo
As terapias empregadas no tratamento de doenças do sistema nervoso central são complexas e em sua maioria trazem inúmeros efeitos adversos, levando a indústria farmacêutica a pesquisar sobre novas substâncias e produzir medicamentos mais eficazes e menos agressivos ao paciente, com o objetivo de estudar a eficácia do canabidiol no tratamento de convulsões epiléticas e demais doenças do sistema nervoso central. $\mathrm{O}$ método adotado foi uma pesquisa exploratória qualitativa, realizada através de busca ativa nas bases de dados do PUBMED, LILACS, SCIELO e em sites oficiais como ANVISA, Liga Brasileira de Epilepsia (LBE) entre outros, abordando epilepsia, canabidiol e convulsões. Os resultados mostraram que, dos artigos selecionados, todos concordam que o canabidiol (CBD) possui ação anticonvulsivante na epilepsia. Porém, houve discordância quanto aos mecanismos de ação e segurança no uso. Destes, alguns explicam suas propriedades agonistas nos receptores 5-HT1A e apontam o CBD como elemento ativador do receptor vanilóide tipo 1. As propriedades terapêuticas do CBD precisam de mais pesquisas para: ampliar o conhecimento sobre essa substância, identificar sua janela terapêutica e conhecer melhor seu mecanismo de ação no tratamento de doenças neurológicas. Gerando maior segurança de administração do fármaco para pacientes e prescritores.
\end{abstract}

Palavras-chave: Cannabis sativa, características dos canabinóides, substâncias anticonvulsivantes, usos terapêuticos.

\section{Efficacy of cannabidiol in the treatment of convulsions and diseases of the central nervous system: systematic review}

\begin{abstract}
The therapies used to treat central nervous system diseases are complex and most of them have many adverse effects, leading the pharmaceutical industry to research new substances and produce drugs that are more effective and less aggressive to the patient. This research studied the efficacy of cannabidiol in the treatment of epilepsy convulsions and other diseases of the central nervous system. The method adopted was a qualitative exploratory research, carried out through active search in the PUBMED, LILACS, SCIELO databases and in official sites such as ANVISA, Brazilian League of Epilepsy and among others, addressing epilepsy, cannabidiol and convulsions. The results showed that, of the articles selected, all agree that cannabidiol (CBD) has anticonvulsive action in epilepsy. However, there was disagreement as to the mechanisms of action and safety in the use. Of these, some explain their agonist properties at 5-HT 1A receptors and point to CBD as activating element of type 1 vanilloid receptor. Most works express the need to elucidate their functioning in the body. The therapeutic properties of CBD need further research to: increase knowledge about this substance, identify its therapeutic window and better understand its mechanism of action in the treatment of neurological diseases. Induce greater safety of administration of the drug for patients and prescribers.
\end{abstract}

Keywords: Anticonvulsant substances, Cannabis sativa, characteristics of cannabinoids, therapeutic uses.

\section{Introdução}

Atualmente, as doenças do sistema nervoso (como o Acidente Vascular Encefálico, Epilepsia, Doença de Parkinson, Doença de Alzheimer) são alvos de terapias complexas que envolvem medicações com variados efeitos adversos e, por isso, a indústria farmacêutica está sempre à procura de novas substâncias e medicamentos mais efetivos e menos agressivos ao organismo do paciente. (Passos, Arbo, Rates \& Poster, 2009).
A epilepsia caracteriza-se por ser uma doença neurológica crônica, geralmente progressiva, gerando alterações cognitivas de acordo com a frequência e gravidade dos eventos críticos, chamados de crises convulsivas (Aspesi \& Perla, 2016). Quanto mais repetidas e intensas forem as convulsões, mais grave será o prognóstico do paciente.

Nesse intuito, a ciência tem voltado suas atenções para 
vegetais com efeitos farmacológicos comprovados, mas que, até então, estavam marginalizados das pesquisas centrais como o caso de Cannabis sativa Lam. Estudos de Coorte e intervencionais comprovam que o canabidiol, extraído de $C$. sativa, gera menor incidência de convulsões e maior conforto para pacientes epiléticos, pois possibilita um sono mais prolongado e eficaz, além de ser um composto sem efeito psicotrópico. (Brucki et al.,2015)

$\mathrm{O}$ CBD age em diversos sistemas neuronais e também no sistema endocanabinóide (GABA, serotonina, glutamato, entre outros). O CBD exerce uma ampla variedade de ação farmacológica sem interação com o sistema dopaminérgico e, portanto, não causando euforia, agitação ou eventos motores quaisquer (Casa da Ciência, 2015).

Em dezembro de 2013, a Food and Drug Administration (FDA) aprovou, nos Estados Unidos, o uso terapêutico do canabidiol em pesquisas para tratamento de epilepsias refratárias em crianças que não reagiram aos tratamentos convencionais. No Brasil, a aprovação norte-americana e êxito de alguns estudos influenciaram na autorização do uso medicinal e industrialização de medicamentos à base de CBD e Tetraidrocanabinol (THC) pela ANVISA (Agência Nacional de Vigilância Sanitária [ANVISA], 2016). Este poderá ser o início de futuras pesquisas sobre outros canabinóides, ainda não analisados em suas propriedades e potenciais efeitos terapêuticos.

O presente estudo é uma revisão de literatura, a relevância está em sua produção ocorrer após a aprovação do uso do CBD e do THC em território brasileiro pela ANVISA. Dessa forma, este trabalho teve, à sua disposição, um maior número de pesquisas produzidas nos últimos anos como fonte de dados. Assim sendo, temos como objetivo evidenciar a ampla aplicabilidade do CBD, averiguando os efeitos farmacológicos, toxicológicos e os mecanismos dessa substância no tratamento de convulsões, epilepsia e demais doenças do sistema nervoso central. Apresentando a utilização do composto como alternativa terapêutica viável na atenuação dos eventos mecânicos e psíquicos envolvidos na problemática da doença.

\section{Material e Métodos}

A coleta de dados ocorreu entre setembro de 2016 e novembro de 2018. Utilizando-se os descritores canabidiol, $C$. sativa, epilepsia, convulsão e seus equivalentes em inglês, foram rastreados artigos, de 2011 a 2018, que tivessem as palavraschave em seu título ou resumo. Além disso, os trabalhos selecionados foram somente aqueles publicados até novembro de 2018 nas bases de dados eletrônicas PUBMED, LILACS, SCIELO e em sites oficiais como ANVISA, LBE entre outros.

Adotaram-se os seguintes critérios de inclusão: artigos publicados nos últimos sete anos e estudos, livros, dissertações e teses sobre epilepsia e comparativos de ação farmacológica com o THC. Posteriormente, percebe-se que o número de publicações, neste intervalo de tempo, foi pequeno e com muitas publicações de revisão sistemática de literatura. Somente foram inclusos os artigos publicados nos idiomas inglês e português entre 2011 e novembro de 2018. Tais publicações foram organizadas com uso de uma tabela de análise.

Foram excluídos os artigos que utilizaram a Cannabis fumada pelo fato de não ser possível estabelecer a dose, a composição e a razão dos diferentes componentes canabinóides. Isto porque há uma ampla variação da concentração dos insumos ativos nas diferentes amostras do vegetal. Foram excluídas também as publicações com mais de sete anos e trabalhos em francês. Para critério de análise textual, foi utilizado questões norteadoras, onde era verificado se o problema e a metodologia estavam, de fato, claro, se os objetivos possuíam relação com a questão que estava sendo estudada, se os resultados obtidos de cada artigo possuíam credibilidade e estavam de acordo com a metodologia, além de verificar a profundidade das pesquisas excluindo as que foram consideradas rasas, sem fundamentos teóricos e que não tinham argumentos consideráveis.

Foram identificados 126 artigos, sendo 102 através da pesquisa dos bancos de dados e 24 de outras fontes. Dentre os 126, houveram exclusões devido o ano de publicação (34), metodologia não condizente com os resultados (39), língua em que o artigo foi publicado (21) e pesquisas onde a profundidade não era adequada (19). Deste modo, 13 artigos foram incluídos na síntese quantitativa.

\section{Resultados e Discussão}

Foram abordadas pesquisas enfatizando o efeito neuroprotetor do canabidiol e a eficácia do medicamento Sativex (98\% de CBD), tanto no tratamento de usuários de opióides, como no tratamento de dependência da maconha. Também foram incluídos estudos de resposta emocional e cognitiva após o uso de THC e CBD, visando esclarecer o efeito antagônico entre os componentes.

As metodologias dos trabalhos avaliados são bem variadas, a maioria aborda testes em humanos (Devinsky, et al., 2014), já outros autores, optaram por testes em ratos Wistar, visando comprovar a eficácia do canabidiol e seus efeitos no sistema nervoso central (Fagherazzi, 2011).

Dos 13 artigos selecionados, todos concordaram que o canabidiol possui ação anticonvulsivante na epilepsia. Porém, houve discordância entre eles quanto aos mecanismos de ação e segurança no uso. De todos os trabalhos, apenas quatro, cujas pesquisas foram feitas em 2011, 2014, 2016 e 2018 explicaram o seu modo de ação através das propriedades agonistas aos receptores 5-HT1A e, que o CBD também ativa o receptor vanilóide tipo 1 (TRPV1). Em contrapartida, dez pesquisas expressaram a real necessidade de elucidação do seu mecanismo. Provavelmente por isso, houve baixa quantidade de artigos científicos publicados sobre o uso do canabidiol como anticonvulsivante, uma vez que se trata de uma metodologia enraizada na experimentação e estudos aprofundados.

As pesquisas reportaram que o CBD não possui efeitos psicoativos e também apresenta amplo espectro de ação farmacológica (Schier et al., 2012). Possui comprovado efeito antiepilético, porém com alguns pontos não bem esclarecidos, como: segurança de administração por longo espaço de tempo, propriedades farmacocinéticas, seu mecanismo de ação e interação farmacológica com outros canabinóides (Brucki et al., 2015). Contudo, é importante considerar que o composto deve ser usado com prudência em pessoas em desenvolvimento cognitivo, como crianças e adolescetes (Devinsky et al., 2014). 
Tabela 2. Resultados dos artigos utilizados na metanálise da pesquisa bibliográfica (2011-2018).

\begin{tabular}{|c|c|c|c|c|c|c|}
\hline Referência & Tipo De Estudo & Amostra & Protocolo & $\begin{array}{c}\text { Intervalo de } \\
\text { Tempo }\end{array}$ & $\begin{array}{c}\text { Efeitos } \\
\text { Adversos }\end{array}$ & Desfecho \\
\hline $\begin{array}{l}\text { Devinsky } \\
\text { et al. } \\
(2014)\end{array}$ & $\begin{array}{l}\text { Teste de eficácia } \\
\text { de medicamento. }\end{array}$ & $\begin{array}{c}37 \\
\text { Pacientes }\end{array}$ & $26 \mathrm{mg} / \mathrm{kg} / \mathrm{dia}$ & 12 semanas & $\begin{array}{l}\text { Sonolência } \\
\text { e fadiga }\end{array}$ & $\begin{array}{c}\text { A segurança e eficácia do } \\
\text { CBD necessitam ser melhor } \\
\text { delineadas. }\end{array}$ \\
\hline $\begin{array}{l}\text { Trigo et al. } \\
\text { (2016) }\end{array}$ & $\begin{array}{l}\text { Administração de } \\
\text { placebo duplo- } \\
\text { cego (design } \\
\text { ABACADAE). }\end{array}$ & $\begin{array}{c}9 \\
\text { pacientes }\end{array}$ & $\begin{array}{c}108,0 \mathrm{mg} \\
\text { THC/100,0 mg } \\
\text { CBD }\end{array}$ & 12 semanas & Ausente & $\begin{array}{l}\text { Evidenciar a eficácia do } \\
\text { medicamento sativex. }\end{array}$ \\
\hline $\begin{array}{l}\text { Manini et } \\
\text { al. }(2015)\end{array}$ & $\begin{array}{l}\text { Testar o } \\
\text { canabidiol em } \\
\text { usuários de } \\
\text { opióides. }\end{array}$ & $\begin{array}{c}17 \\
\text { pacientes }\end{array}$ & $\begin{array}{c}0,5 \mathrm{mcg} / \mathrm{kg} \\
\text { fentanil } \\
1,0 \mathrm{mcg} / \mathrm{kg} \mathrm{CBD}\end{array}$ & 9 semanas & $\begin{array}{c}\text { Não } \\
\text { significativo }\end{array}$ & $\begin{array}{l}\text { Não satisfatório devido à } \\
\text { similaridade entre os efeitos } \\
\text { das drogas utilizadas. }\end{array}$ \\
\hline $\begin{array}{l}\text { Karschner } \\
\text { et al. } \\
(2014)\end{array}$ & $\begin{array}{l}\text { Testes em } \\
\text { humanos. }\end{array}$ & $\begin{array}{l}22 \\
\text { pacientes } \\
\text { sadios }\end{array}$ & $\begin{array}{l}\text { (5,4 mg de THC, } \\
\text { 5,0 mg de CBD) }\end{array}$ & $\begin{array}{l}1,0,3,0,4,5 \text { e } \\
5,5 \text { h após a } \\
\text { dose. }\end{array}$ & $\begin{array}{l}\text { Aumento da } \\
\text { pressão } \\
\text { arterial }\end{array}$ & $\begin{array}{l}\text { Não conclusivo, sem } \\
\text { parâmetro de custo } \\
\text { benefício. }\end{array}$ \\
\hline $\begin{array}{l}\text { Fagherazzi } \\
\text { (2011) }\end{array}$ & $\begin{array}{l}\text { Modelo animal de } \\
\text { dano cognitivo } \\
\text { induzido. }\end{array}$ & $\begin{array}{l}60 \text { ratos } \\
\text { machos } \\
\text { Wistar. }\end{array}$ & $\begin{array}{c}\text { Grupo 01: } 5 \\
\text { mg/kg CBD + } 10 \\
\text { mg/ kg Fe } \\
\text { Grupo 02: } 10 \\
\mathrm{mg} / \mathrm{kg} \mathrm{CBD} \mathrm{+} 10 \\
\mathrm{mg} / \mathrm{kg} \mathrm{Fe}\end{array}$ & 60 dias & Ausente & $\begin{array}{l}\text { Melhora significativa na } \\
\text { perda de memória induzida } \\
\text { nos ratos. }\end{array}$ \\
\hline $\begin{array}{l}\text { Hindocha, } \\
\text { Chandni et } \\
\text { al., (2015) }\end{array}$ & $\begin{array}{l}\text { Teste de resposta } \\
\text { emocional }\end{array}$ & $\begin{array}{c}48 \\
\text { pacientes }\end{array}$ & $8 \mathrm{mg}$ CBD & 4 dias & $\begin{array}{c}\text { Não } \\
\text { significativo }\end{array}$ & $\begin{array}{l}\text { O CBD modulou as } \\
\text { respostas emocionais } \\
\text { induzidas pelo THC. }\end{array}$ \\
\hline $\begin{array}{l}\text { Szaflarski } \\
\text { et al. } \\
(2018)\end{array}$ & $\begin{array}{l}\text { Teste de segurança } \\
\text { e efetividade }\end{array}$ & $\begin{array}{c}607 \\
\text { pacientes }\end{array}$ & $\begin{array}{c}\text { Começando } 2 \text { - } \\
10 \mathrm{mg} / \mathrm{kg} / \mathrm{dia} \\
\text { Tentando dose } \\
\text { máxima } 25 \text { - } 50 \\
\mathrm{mg} / \mathrm{kg} / \text { dia }\end{array}$ & 96 semanas & $\begin{array}{l}\text { Diarreia e } \\
\text { sonolência }\end{array}$ & $\begin{array}{l}\text { CBD pode ser de grande } \\
\text { eficácia em uso prolongado } \\
\text { e mostrou que é uma droga } \\
\text { com boa tolerância }\end{array}$ \\
\hline $\begin{array}{l}\text { Rosenberg, } \\
\text { Louik, } \\
\text { Conway, } \\
\text { Devinsky, } \\
\text { Friedman, } \\
(2017)\end{array}$ & $\begin{array}{l}\text { Medir a melhoria } \\
\text { da qualidade de } \\
\text { vida dos pacientes }\end{array}$ & $\begin{array}{c}48 \\
\text { pacientes }\end{array}$ & $25 \mathrm{mg} / \mathrm{kg} \mathrm{CBD}$ & 12 semanas & $\begin{array}{l}\text { Sonolência } \\
\text { e fadiga }\end{array}$ & $\begin{array}{l}\text { Houve uma melhora na } \\
\text { qualidade de vida } \\
\text { significante dos pacientes }\end{array}$ \\
\hline $\begin{array}{l}\text { Suraev et } \\
\text { al. }(2018)\end{array}$ & $\begin{array}{c}\text { Verificar a } \\
\text { efetividade do } \\
\text { CBD na infancia }\end{array}$ & $\begin{array}{c}51 \\
\text { crianças }\end{array}$ & $\begin{array}{l}0.01-1.23 \\
\mathrm{mg} / \mathrm{kg} / \mathrm{dia}\end{array}$ & 13 semanas & $\begin{array}{l}\text { Aumento de } \\
\text { convulsões } \\
\text { em alguns } \\
\text { pacientes }\end{array}$ & Redução das convulsões \\
\hline $\begin{array}{l}\text { Mccoy et } \\
\text { al. (2018) }\end{array}$ & $\begin{array}{c}\text { Teste de } \\
\text { tolerabilidade } \\
\text { eventos adversos } \\
\text { em crianças }\end{array}$ & $\begin{array}{c}20 \\
\text { pacientes }\end{array}$ & $\begin{array}{c}2-16 \mathrm{mg} / \mathrm{kg} / \mathrm{dia} \\
\mathrm{CBD} \\
0,04-0,32 \\
\mathrm{mg} / \mathrm{kg} / \mathrm{dia} \text { THC }\end{array}$ & $\begin{array}{l}20 \text { semanas, } \\
\text { aumentando a } \\
\text { dose } \\
\text { semanalmente }\end{array}$ & Sonolência & $\begin{array}{c}\text { Segurança e tolerabilidade } \\
\text { satisfatória. }\end{array}$ \\
\hline $\begin{array}{l}\text { Szaflarski } \\
\text { et al. } \\
(2018)\end{array}$ & $\begin{array}{l}\text { Verificação de } \\
\text { convulsões com } \\
\text { CBD }\end{array}$ & $\begin{array}{c}139 \\
\text { pacientes }\end{array}$ & $25 \mathrm{mg} / \mathrm{kg} / \mathrm{dia}$ & 48 semanas & Ausente & $\begin{array}{l}\text { Diminuição da intensidade } \\
\text { e frequência das convulsões }\end{array}$ \\
\hline $\begin{array}{l}\text { Rosenberg, } \\
\text { Tsien, } \\
\text { Whalley, } \\
\text { Devinsky, } \\
2015\end{array}$ & $\begin{array}{l}\text { Revisão dados } \\
\text { recentes de } \\
\text { estudos clínicos }\end{array}$ & 17 estudos & $\begin{array}{c}100-300 \mathrm{mg} / \mathrm{dia} \\
\mathrm{CBD}\end{array}$ & $\begin{array}{c}3 \text { semanas a } 6 \\
\text { meses }\end{array}$ & Sonolência & Diminuição das convulsões \\
\hline $\begin{array}{l}\text { Reddy e } \\
\text { Golub et } \\
\text { al., (2015) }\end{array}$ & $\begin{array}{l}\text { Verificação de } \\
\text { redução da } \\
\text { frequência e } \\
\text { duração de } \\
\text { convulsões }\end{array}$ & 15 estudos & $\begin{array}{l}4-300 \mathrm{mg} / \mathrm{dia} \\
\mathrm{CBD}\end{array}$ & $\begin{array}{l}4 \text { semanas a } \\
12 \text { meses }\end{array}$ & $\begin{array}{l}\text { Sonolência, } \\
\text { fadiga, } \\
\text { diarreia e } \\
\text { perda de } \\
\text { peso }\end{array}$ & $\begin{array}{l}\text { Redução da frequência e da } \\
\text { duração das convulsões }\end{array}$ \\
\hline
\end{tabular}

Os compostos canabinóides geram novas pesquisas devido ao fato da química da Cannabis ser muito complexa e ainda não ser totalmente compreendida, além das controvérsias relacionadas ao efeito colateral próprio de cada substância encontrada na planta (Honório, Arroio \& Silva, 2005). Nela, o CBD e o THC são os seus compostos mais presentes.

A utilização do CBD no tratamento de desmame de 
usuários de drogas, que às vezes gera convulsões, mostra-se eficaz e mais seguro do que algumas medicações para este fim. Essa substância tem sido testada na abstinência da Cannabis fumada (Trigo et al., 2016) e, até em inveterados em opióides (Manini et al., 2015) com resultados favoráveis e bastante promissores.

Além da ampla atuação do CBD sobre as disfunções do sistema nervoso central (SNC), alguns estudos indicam que tanto o canabidiol quanto os extratos de $C$. sativa foram capazes também de impedir a proliferação de células cancerígenas. A apoptose foi induzida pelo canabidiol, demonstrando seu poder antineoplásico (Lukhele \& Motadi, 2016).

O THC age no corpo humano pela ligação com seus receptores específicos $\mathrm{CB} 1$ (receptor canabinoide tipo 1) e CB2 (receptor canabinoide tipo 2) presentes no sistema nervoso central. Os receptores CB1 estão presentes tanto em neurônios inibitórios gabaérgicos quanto em neurônios excitatórios glutamatérgicos, ambos na membrana présináptica destas células (Devinsky et al., 2014).

A interação do THC com receptores específicos já é conhecida desde a década de 60. Os receptores CB1 estão diretamente ligados ao sistema nervoso central, enquanto os CB2 estão conectados ao sistema imunológico (Honório et al., 2005), diferindo do modo de ação do canabidiol que parece ter pouquíssima atividade em relação a estes receptores. Logo, o canabidiol apresenta características neuroprotetoras e ação anticonvulsivante, diferente do THC.

$\mathrm{O}$ efeito anticonvulsivante do canabidiol é promissor, justificando a sua inclusão no arsenal terapêutico porque possui efeito antiepiléptico reconhecido. Para isto, a cada ano estão sendo realizados novos testes de ensaio clínicos com metodologias variadas, que pretendem elucidar tudo aquilo que se mantém vago, no que tange à segurança na utilização do composto (Bonfá, Vinagre \& Figueredo, 2008).

A Food and Drug Administration (FDA) tem sido uma das pioneiras em estudos aprofundados sobre $\mathrm{O}$ efeito antiepilético do CBD. Um trabalho multicêntrico, desenvolvido em 11 centros especializados nos EUA (Estados Unidos da América), foi liderado por Devinsky et al. (2014) que mostrou, além da eficácia do óleo no tratamento das convulsões, alguns efeitos adversos pouco esclarecidos devido ao porte menor de estudos anteriores. Alguns exemplos de tais condições são: sonolência, fadiga, perda ou ganho de peso, diarreia e aumento ou redução do apetite. Isso mostra a necessidade de avançar ainda mais na profundidade dos trabalhos para estabelecer os limites terapêuticos da droga.

Os avanços das pesquisas são alcançados com diferentes metodologias como duplo-cegos e experimentos com ratos Wistar. Alguns estudos duplo-cegos abordados têm mostrado efeitos variados em relação ao uso do canabidiol no tratamento de doenças do SNC, como epilepsia, Parkinson e autismo. Os experimentos com tais ratos se mostraram bastante promissores, no que se refere à neuroproteção, desde que seja feita a utilização do canabidiol precocemente. $\mathrm{Na}$ recuperação de cobaias sem o prévio uso do composto, os resultados são bastante variáveis, alguns até ineficazes (Fagherazzi, 2011).
O canabidiol induz os seus efeitos farmacológicos sem exercer qualquer atividade intrínseca significativa sobre os receptores canabinóides, cuja ativação resultaria em efeitos psicotrópicos, como ocorre com o $\Delta$ (9) THC. O CBD possui várias atividades farmacológicas que lhe conferem um elevado potencial para utilização terapêutica agindo como: neuroprotetor, antiepilépticos, ansiolíticos e antipsicóticos, além de apresentar propriedades anti-inflamatórias e antitumorais (Crippa et al, 2005).

Provavelmente, o canabidiol age modulando a transmissão sináptica por bloqueio dos canais de cálcio $(\mathrm{Ca} 2+)$ e potássio $(\mathrm{K}+)$ dependentes de voltagem. É possível que, desta forma, o canabidiol iniba as crises epiléticas e convulsões, evitando a superexcitação das transmissões neuronais. (Porto et al., 2007). É notável a falta de consenso sobre este assunto, ainda dividindo a opinião de alguns especialistas.

Outras metodologias têm enfatizado os efeitos contrários do CBD em relação ao THC como ferramenta de pesquisa, para elaborar as doses efetivas, a janela terapêutica e, em parte, elucidar o mecanismo de ação do canabidiol (Fusar-Poli et al., 2009). Demonstra-se que vários efeitos causados pelo THC, como letargia, falha na memória curta e dependência química, são revertidos em pacientes que fizeram uso do CBD.

O efeito anticonvulsivante, por si só, justifica novos estudos acerca do canabidiol, pois a maioria dos testes de ensaios clínicos aponta que o CDB se tornará uma das principais alternativas terapêuticas para o controle e tratamento desses eventos convulsivos em pacientes com epilepsia (Bonfá et al., 2008). Devido a isto, nos últimos anos, ocorre uma atualização na legislação brasileira propiciando o desenvolvimento de mais pesquisas sobre o uso terapêutico desta substância (ANVISA, 2016).

Contudo, o CBD exige prudência em sua administração para evitar comprometimento maior na saúde do paciente. Segundo Crippa et al. (2005), os efeitos do canabidiol são bifásicos, atuando na ativação de áreas paralímbicas e límbicas de forma semelhante a outros ansiolíticos. Portanto, é complicado estabelecer a faixa terapêutica do fármaco, o que tornam necessárias novas pesquisas.

As fontes de pesquisas e o número de trabalhos efetivos ainda são poucos, devido à legislação de muitos países ainda considerar os subprodutos da Cannabis como drogas ilícitas e, portanto, marginalizando os compostos, barrando os estudos e inviabilizando-os como ferramentas terapêuticas. Isso justifica o grande número de revisões sistemáticas de estudos primários na maioria dos países, as exceções são grandes centros de pesquisas como Canadá e Estados Unidos.

Portanto, com as devidas modificações na legislação brasileira, nossos centros de pesquisa produzirão um maior número de trabalhos sobre o CBD. Desta maneira, serão acelerados os resultados sobre o canabidiol, proporcionando seu uso de forma segura e eficaz, aumentando as alternativas terapêuticas e impulsionando a indústria farmacêutica na elucidação de outros compostos canabinóides ainda não explorados.

\section{Conclusões}

As informações apresentadas, nesta revisão, indicam o canabidiol como potencialmente apto para ser incluído no 
arsenal terapêutico, porém os estudos de até então não elucidam o seu mecanismo de ação, nem se mostram seguros sobre a utilização da substância por período prolongado. Isto porque, no tratamento epilético, a maior parte dos acometidos é composta de jovens, ainda em período de desenvolvimento cognitivo.

Portanto, mais pesquisas sobre o canabidiol devem ser realizadas para elucidar seu mecanismo de ação no organismo humano, gerando maior segurança na administração de uso para pacientes, cuidadores e prescritores.

\section{Referências}

Agência Nacional de Vigilância Sanitária. (2016). Canabidiol e THC: norma permitirá registro de produto. Brasília: Agência Nacional de Vigilância Sanitária, Brasília.

Aspesi, N. V. \& Perla, A. S. Epilepsia, convulsão e ataque epiléptico. Recuperado de: https://www.abcdasaude.com.br/neurologia/epilepsiaconvulsao-ataqueepileptico.

Bonfá, L., Vinagre, R. C. O. \& Figueredo, N. V. (2008). Uso de Canabinóides na Dor Crônica e em Cuidados Paliativos. Revista Brasileira Anestesiologia, 58(3), 267-279. doi: 10.1590/S003470942008000300010

Brucki, S. M. D., Frota, N. A., Schestatsky, P., Souza, A. H., Carvalho, V. N., Manreza, M.L.G., ... Jurno, M. E. (2015). Canabinoides e seu uso em neurologia. Arquivos Neuro-Psiquiatria, 73(4), 371-374. doi: 10.1590/0004-282X20150041

Casa da ciência, (2015). Os canabinóides e seu cérebro. Hemocentro de Ribeirão Preto - FMRP-USP. Recuperado de: ead.hemocentro.fmrp.usp.br/joomla/index.php/noticias/adotepauta/639os-canabinoides-e-seu-cerebro.

Crippa, J. A., Lacerda, A. L. T., Amaro, E., Busatto-Filho, G., Zuardi, A. W. \& Bressan, R. A. (2005). Efeitos cerebrais da maconha: resultados dos estudos de neuroimagem. Revista Brasileira de Psiquiatria, 27(1), 7078. doi: 10.1590/S1516-44462005000100016

Devinsky, O., Cilio, M. R., Cross, H., Fernandez-Ruiz, J., French, J., Hill, C., ... Friedman, D. (2014). Cannabidiol: Pharmacology and potential therapeutic role in epilepsy and other neuropsychiatric disorders. Wiley periodicals, 55(6), 791-802. doi: 10.1111/epi.12631

Fagherazzi, E. V. (2011). Uso do Canabidiol como protetor contra disfunções cognitivas associadas ao acúmulo de ferro cerebral em ratos Wistar (Dissertação de Mestrado). Pontifícia Universidade Católica do Rio Grande do Sul de Biociências, Porto Alegre, Rio Grande do Sul, Brasil.

Fusar-Poli, P., Crippa, J. A., Bhattacharyya, S., Borgwardt, S. J., Allen, P. \& Martin-Santos, R. (2009). Distinct Effects of $\Delta 9$ Tetrahydrocannabinol and Cannabidiol on Neural Activation During Emotional Processing. Archives of general Psychiatry, 66(1), 95-105. doi: 10.1001/archgenpsychiatry.2008.519

Hindocha, C., Freeman, T. P., Schafer, G., Gardener, C., Das, R. K., Morgan, C. J. \& Curran, H. V. (2015). Efeitos agudos do delta-9tetrahidrocannabinol, canabidiol e sua combinação no reconhecimento da emoção facial: Estudo randomizado, duplo-cego, controlado por placebo em usuários de Cannabis. European Neuropsychopharmacol, 25(3), 325-334. doi: 10.1016/j.euroneuro.2014.11.014.

Honório, K. M., Arroio, A. \& Silva, A. B. F. (2005). Aspectos terapêuticos de compostos da planta Cannabis sativa. Química Nova, 29(2), 318-325. doi: 10.1590/S0100-40422006000200024

Karschner, E. L., Darwin, W. D., McMahon, R. P., Liu, F., Wright, S., Goodwin, R. S., Huestis, M. A. (2011). Subjective and Physiological Effects After Controlled Sativex and Oral THC Administration. Clinical pharmacology e therapeutics, 89(3), 400-407. doi: $10.1038 /$ clpt.2010.318

Lukhele, S. T. \& Motadi, L. R. (2016). Cannabidiol rather than Cannabis sativa extracts inhibit cell growth and induce apoptosis in cervical cancer cells. BMC Complementary and Alternative Medicine, 16(1), 335. doi: 10.1186/s12906-016-1280-0

Manini, A. F., Yiannoulos, G., Bergamaschi, M. M., Hernandez, S., Olmedo,
R., Barnes, ... Hurd, Y. L. (2015). Safety and pharmacokinetics of oral cannabidiol when administered concomitantly with intravenous fentanyl in humans. Journal of Addiction Medicine, 9(3), 204-210. doi: 10.1097/ADM.0000000000000118

McCoy, B., Wang, L., Zak, M., Al-Mehmadi, S., Kabir, N., Alhadid, K., ... Snead III, O. C. (2018). A prospective open-label trial of a CBD/THC cannabis oil in dravet syndrome. Annals of Clinical and Translational Neurology, 5, 1077-1088. doi: 10.1002/acn3.621

Passos, C. S., Arbo, M. D., Rates, S. M. K. \& Poser, G. L. V. (2009). Terpenóides com atividade sobre o Sistema Nervoso Central (SNC). Revista Brasileira de Farmacognosia, 19(1), 140-149. doi: 10.1590/S0102-695X2009000100024

Porto, L. A., Siqueira, J. S., Seixas, L. N., Almeida, J. R. G. S. \& QuintansJunior, L. J. (2007). O papel dos Canais Iônicos nas Epilepsias e Considerações Sobre as Drogas Antiepilépticas: uma breve revisão. Journal Epilepsy Clin. Neurophysiology, 13(4), 169-175. doi: 10.1590/S1676-26492007000400005

Reddy, D. S. \& Golub, V. M. (2016). The pharmacological basis of cannabis therapy for epilepsy. The journal of pharmacology and experimental therapeutics, 357, 45-55. doi: 10.1124/jpet.115.230151

Rosenberg, E. C., Tsien, R. W., Whalley, B. J. \& Devinsky, O. (2015). Cannabinoids and epilepsy. Neurotherapeutics, 12(4), 747-768. doi: 10.1007/s 13311-015-0375-5

Rosenberg, E. C., Louik, J., Conway, E., Devinsky, O. \& Friedman, D. (2017). Quality of life of childhood epilepsy on pediatric enrolled in a prospective, open-label clinical study with cannabidiol. Official journal of the international league against epilepsy, 58(8), 96-100. doi: 10.1111/epi.13815

Schier, A. R. M., Ribeiro, N. P. O., Silva, A. C. O., Hallak, J. E. C., Crippa, J. A. S., Nardi, A. E. \& Zuardi, A. W. (2012). Canabidiol, um componente da Cannabis sativa, como um ansiolítico. Revista Brasileira de Psiquiatria, 34(1), 104-117. doi: 10.1590/S1516-44462012000500008

Suraev, A., Lintzeris, N., Stuart, J., Kevin, R. C., Blackburn, R., Richards, ... McGregor, I. S. (2018). Composition and use of cannabis extracts for childhood epilepsy in the australian community. Scientific Reports, 8 , 10154. doi: 10.1038/s41598-018-28127-0

Szaflarski, J. P., Bebin, E. M., Comi, A. M., Patel, A. D., Joshi, C., Checkets, D., Beal, ... Wechsle, R. (2018). Long-term safety and treatment effects of cannabidiol in children and adults with treatment-resistant epilepsies, Expanded access program results, Epilepsia, 59, 1540-1548. doi: 10.1111/epi.14477

Szaflarski, J. P., Bebin, E. M., Cutter, G., DeWolfe, J., Dure, L. S., Gaston, T. E., ... Hoef, L. W. V. (2018). Cannabidiol improves frequency and severity of seizures and reduces adverse events in an open-label add-on prospective study. Epilepsy and behavior, 87, 131-136. doi: 10.1016/j.yebeh.2018.07.020

Trigo, J. M., Lagzdins, D., Rehm, J., Selby, P., Gamaleddin, I., Fischer, B., ... Foll, B. L. (2016). Effects of fixed or self-titrated dosages of Sativex on cannabis users. Drug Alcohol Depend, 161(1), 298-306. doi: 10.1016/j.drugalcdep.2016.02.020

\section{Licença Creative Commons CC BY 4.0}

Este artigo foi publicado com acesso aberto para distribuíção sob os termos do Licença de Atribuição Creative Commons, que permite uso irrestrito, distribuição, e reprodução em qualquer meio, desde que o trabalho original seja devidamente citado. 